\title{
Perspectives of the European academic research in the microwave and millimeter wave area
}

\author{
Angel Cardama \\ Polytechnic University of Catalonia, P.O. Box 30002, 0807 I- \\ Barcelona, Spain
}

When the Chairman invited me to present this talk on our future perspectives, he pointed out I should consider emphasizing the general aspects, leaving out the more detailed technical features. I was at first concerned by the difficulties of addressing those issues, instead of the more narrow technical ones, that we are all more familiar with, and feel more comfortable presenting. Given that the conference program has an in-depth coverage of the most recent and relevant work performed in the field, and we have a sizeable number of invited papers that will give you a tutorial perspective of the topics of present interest, I hope not to disappoint many of you by bringing up some points more frequently debated around a cup of coffee than in the conference floor.

I will take the view of considering only the European academic community, giving you some candid personal opinions and trying not to exhaust the time allowed, so some of you in the audience could bring up different comments or opinions, and argue differently on this subject.

I feel it is appropriate to bring forward this matter at this time, when we have a very successful conference in terms of attendance and technical quality of the contributions and, at the same time, one can perceive a general atmosphere of economic, social and industrial crisis. It is in those moments, when one is at a crossroad, that the possible paths to follow should be considered and its difficulties assessed.

Let me start by asking some key questions on aspects that I believe will greatly influence our future:

* Are we a well established community, working in a field with most of its groundwork already done and its research tools well known?

Is there any room for future breakthroughs?

Will they occur in other related fields with little participation and benefit on our part?

Where are they most likely to appear?

In what areas will our technology be applied to solve existing or new problems, or to satisfy social needs or demands?

* Is our an aging field where we can only foresee minor growth?

If so, how should we position ourselves, both individually and as a group?

* Is the size of our community appropriate?

Is it oversized or there is still room for growth?

Is there any real academic future for our young researchers and graduate students?

Is this a field they should enter confidently or will they be better off pursuing a career elsewhere?

* What will be the impact of the European Community policy regarding research and development funding, and researchers mobility?

Will it have an strong impact or national policies will continue to set the trends of our research efforts?

This is a sample of what I feel are some of the questions that many of us ask ourselves quite frequently, especially in very hard times. I will try to comment on some of them, for others, I honestly do not have a clear answer, and its perception could be quite different depending on country, university or research group.

From a very general point of view, ours is what we may call a classical field, in the sense this word is used in physics, for example. In many aspects, most of the basics are well established, so we keep increasing the realm of knowledge by building on very solid foundations, with new tools introduced from time to time.

Fortunately, we are a technology driven field, sustained by new devices and systems with a final aim of commercial application. Radio is the backbone of broadcasting, satellite communications, mobile communications, both outdoor and indoor, remote sensing and global positioning, that will support those emerging technologies of wireless personal communications, (imagine calling from anyplace and at anytime!), or intelligent highways and navigation systems. It is evident that research efforts in this area are more and more centered around industries than in academic institutions.

Also, one should not forget that, in most instances, university positions are opened (or closed!) on the basis of teaching loads, although a research profile is in some way always considered. Broadly speaking, two kind of courses can be found in our curricula, very basic core courses, that can be taught by a broad spectrum of faculty members and a highly specialized set of advanced courses taken only by a reduced number of highly motivated, usually very bright students. This tends to make things harder, since, unless there is an strong research activity in the field, EM theory related courses in an institution could very well be taught by a faculty with research interests in a different field. This is a trend that has been going on in some US Universities for many years, even though it has to be said that new successful programs have also been established in others.

Looking into the past, one may find fields where we have maintained a solid grasp, while others have developed very fast in a large community on its own. Examples of the first are computational methods for microwave structures and devices, antennas and guided and non-guided wave propagation, while lightwave communications or adaptive arrays are examples of the last.

Fiber optics communications is an area worth dwelling on for a second, given its past, and the future implications it will continue to have. It, grew up with the very active participation of prominent members of our community, drawing expertise from people with a background on wave and beam propagation, optics, materials sciences, microelectronics and communications. At present, I can see more or less the same kind of situation in the areas of non guided optical communications (inter-satellite links and optical ground stations), quasi-optical techniques for wave beam propagation at millimeter and submillimeter wavelengths, and optical-microwave interactions, all three of them fields that may have a rapid development in the future, and we should explore carefully in search of opportunities.

Looking into another closely related field of activity, the continuous trend in microelectronics towards sizes deep into the submicron region, and multi-gigabit logic, will keep demanding wave-like methods of analysis. In this context, interconnection and packaging problems will continue to require full wave solutions. Also, in present day high capacity communications systems, intermediate frequencies or even baseband signals fall into the microwave region, requiring in its design well known tools of our trade.

Compatibility problems will increase steadily and the need of interference suppression techniques will accompany us in the future. Again, this is a multidisciplinary field where electronagnetics will play an important role.

Going into more closely related fields, antennas is one of the most amazing ones to comment. If Hertz were to revive, he would undoubtedly recognize many of the types of antennas he would be seeing around, and still, it is a burgeoning field with new types of 
antennas coexisting with very old ones, where also a very high degree of refinement in performance evaluation and design has been achieved. Just to show an example, microstrip antennas, an already heavily worked topic, hold a promising future for its low cost, simplicity of fabrication, conformability, and easy integration with circuits. Computed aided design and modelling of the patches and arrays, broadbanding, incorporation of new substrates (superconductors, anisotropic or low-loss materials) and integration with electro-optics and optical fiber devices are areas that will continue to require large research efforts. Antenna integration with transceiver modules in large arrays is another area of great interest.

Adaptive arrays is a topic that has grown mostly outside the radiofrequency community, with most of the work done by signal processing people. There is ample room for work in this field as most of the sensors used are electromagnetic. Besides the classical uses as side lobe cancellers, direction of arrival estimators or adaptive nulling, the great recent advances in the performance of analog-to-digital converters could also foster the use of digital beamforming in communications systems.

Mobile communications have seen an explosive growth in recent years, and propagation models are still the subject of active research, as much as devices and subsystems. An unexpected concern for the effects of radiofrequencies on the human body, as a consequence of the widespread use of portable equipment, has arisen and there is a renewed interest on evaluating the interaction of electromagnetic fields with human tissues. Bioelectromagnetics, a subject that seems to be fading away, is not really doing so, and activity in this area should go on in the future.

Satellite communications systems will always be a highly specialized field, with most of the work directly supported by ESA or industrial companies. Some university groups are maintaining, and will continue to do so in the future, very successful research programs on antennas and beamforming networks.

Remote sensing is an area that will maintain an interest. New systems are been considered and there is a social awareness and a political acceptance of its needs, that can be also extended to all the environmental matters. Microwave systems are now in an early stage, as were optical systems some years ago, and they are expected to reach also widespread use. A somewhat related field, microwave imaging is slowly progressing into industrial applications, as well as microwave power is finding its way into the processing of materials.

Radar will find an extensive use in civilian applications, outside air traffic control and navigation. Any intelligent highway system will depend both on radio for communications and positioning, and on radar for cruising control and collision avoidance. Surveillance systems will also be using microwave technologies, and data fusion from very different sensors is an area widely open.

To sum up and answer some of the starting questions, ours is certainly not an exhausted field, but is the subject of vigorous competition, where no simple problem remain to be solved or groundbreaking technologies are bringing up new easy unsolved questions. Most of our work, either involve complex field operators and require sofisticated numerical codes, or is strongly dependent on technology. This defines a mature field, dynamically growing from well established grounds.

Research has never been an easy task, besides performing it, we are all expert administrators, accountants, negotiators, salesmen, and even typists. On top of that, it might be the time to consider opening up new research lines, without abandoning present day topics. The problems are becoming more and more complex, they are often dependent on available technologies and frequently require an industrial partner. Given that, one should consider entering a new field or at least exploring selectively the topics in related areas where his background or expertise could be applied. As pointed out, in a very general way, there are some open fields of activity in addition to the ones we are all deeply involved with.

I do not see, in the future, many growth opportunities in the academic community. First of all because of demographic reasons, at least in western Europe, secondly because this is a well established field with most of the available positions already filled, and finally because I feel it is oversized in general terms. There is little to comment on the first two reasons. The third one may not be easy to sustain. I have run a very simple test, that it may not be statistically correct, but I think points out in that direction. Taking the full year of 1992 of the IEEE Transactions on MTT and checking the authors with a permanent status, the number from European academic organizations was about $40 \%$ higher than those from north-American institutions, and roughly eight times the number from Japanese universities. Those figures have a very positive reading on the quality of our research groups, but also, if one takes into account the disparity on publication habits, the different national scientific outputs across Europe, and a natural tendency to publish in national journals and symposia, I believe the size of our community is larger than expected and this should be a point of consideration regarding a future academic career in this field.

Let me comment briefly on the European Community policy regarding research and development. As is well known, most of the funding is directed to industrial consortia, that almost always include university partners. The latest workplan of RACE contemplated tasks on personal communications systems, integration of satellites in the future mobile networks, and components for mobile telecommunications systems. The other large Information Technologies program, ESPRIT, contained, in its latest workplan, activities on MMICs and on high performance computers. Those were the only microwave related tasks. By far, the program that will have a wider impact is the human capital and mobility, that is promoting networking between academic institutions and awarding fellowships for the mobility of young graduates. It is open to all fields of sciences and technologies. In all, except for this last program, the community funding will have a minor effect on setting the pace of university research in the microwave field. Scientific guidelines will have to be issued by national bodies and financial support should keep coming from national sources.

There are some research topics that will be sustained by existing resources or that will only require access to computational facilities already available. Many others require external resources and equipment, so they will be dependent on industrial contracts or funding from national agencies. A short list of some topics that will continue to receive support follows.

Automobile and transport applications

Bioelectromagnetics

$\mathrm{CAD}$ and modelling of circuits and components

Computational electromagnetics

Electromagnetic compatibility

MIMIC

Microwave imaging

Microwave industrial applications

Millimeter wave systems

Mobile communications

Navigation and positioning systems

Optical communications

Remote sensing

Satellite communications 